\title{
Multimodal Imaging and Spectroscopy Approaches for Studying Ceramide-induced Reorganization of Lipid Membranes.
}

\author{
Linda J. Johnston ${ }^{1}$, Daniel M. Carter Ramirez ${ }^{1,2}$, Jenna Day ${ }^{1,2}$, Zygmunt Jakubek ${ }^{1}$, Young Ah Kim ${ }^{3}$ and \\ Robert Bittman ${ }^{3}$ \\ ${ }^{1}$ Measurement Science and Standards, National Research Council Canada, Ottawa, ON, K1A 0R6 \\ Canada \\ ${ }^{2}$ Department of Chemistry, University of Ottawa, Ottawa, ON, K1N 6N5 Canada \\ ${ }^{3}$. Department of Chemistry and Biochemistry, City College of the City University of New York, \\ Flushing, NY, USA 11367
}

Multimodal imaging methods that combine multiple contrast mechanisms have been widely applied to study the localization and cooperative rearrangement of membrane lipids and proteins in supported lipid membranes, as a model for the heterogeneity of natural cell membranes. We use a combination of fluorescence spectroscopy and microscopy and atomic force microscopy (AFM) to probe the organization of lipid domains in supported lipid monolayers and bilayers and to examine the distribution of lipids and proteins in these domains. The application of these methods is illustrated with studies of the membrane reorganization promoted by ceramide incorporation. Ceramides are among the most hydrophobic lipids and have distinct effects on the physical properties of membranes, promoting phase separation, non-lamellar phases and membrane permeability [1]. The enzymatic generation of ceramide is believed to cause coalescence of small ordered membrane domains to give larger signaling platforms, thus providing a mechanism to aggregate membrane receptors and enhance signaling efficiency [2]. We have examined the consequences of ceramide incorporation in supported membranes prepared from ternary lipid mixtures that have coexisting fluid and liquid-ordered phases [3]. The direct incorporation of ceramide leads to the formation of a new ceramide-rich ordered phase that is localized in small subdomains within the original ordered domains. Enzymatic generation of ceramide also produces ceramide-enriched islands, but leads to a larger scale reorganization that includes clustering of domains, formation of areas of fluid phase that are devoid of domains and formation of membrane defects.

We have used correlated AFM and fluorescence imaging combined with custom-designed NBD-labeled cholesterol and ceramide probes to provide further information on the complex bilayer restructuring induced by enzymatic generation of ceramide. Images obtained using an NBD-cholesterol probe to examine membrane restructuring are shown in Figure 1 A,B. Enzyme treatment results in the rapid growth of large dye-excluded regions that exclude the cholesterol probe, suggesting that these are ceramide-enriched regions of the membrane. This hypothesis was confirmed by using a ceramide probe with an NBD fluorophore attached to the lipid headgroup (NBD-Cer) for polarized total internal reflection fluorescence microscopy experiments. Measuring fluorescence images as a function of the polarization of the excitation beam can be used to obtain the average fluorophore tilt angle and calculate order parameter maps for bilayers labeled with NBD-Cer. The order parameters provide information on the organization of the bilayer and demonstrate that the large dye-excluded patches formed by enzymatic reaction are more ordered than either the liquid-ordered or liquid-disordered regions of the bilayer, consistent with their assignment to a new ceramide-enriched phase (Figure $1 \mathrm{C}, \mathrm{D}$ ).

Difficulties in controlling and reproducing the morphologies of enzyme-restructured bilayers prompted us to explore the photochemical generation of ceramide using a 6-bromo-7-hydroxycoumarin-caged 
lipid, Bhc-Cer [6]. The photochemical generation of ceramide in phase separated mixtures containing Bhc-Cer results in modification of the liquid-ordered domains, an effect that we hypothesize to be due to the loss of cholesterol from the domains in response to ceramide formation. In fluid phosphatidylcholine (PC) bilayers ceramide generation by photo-uncaging within spatially restricted regions leads to transient formation of ceramide domains (Figure 2) that disappear by equilibration of the ceramide with the surrounding unirradiated bilayer. Additional experiments showed that the photochemical formation of ceramide promotes redistribution of the membrane anchored protein cholera toxin into the ceramide domains. The Bhc-Cer probe is readily taken up by cells, unlike long chain ceramides which typically require use of dodecane for cellular delivery, and releases ceramide efficiently. Overall, the results demonstrate that photo-uncaging of Bhc-Cer generates ceramide with both spatial and temporal control and promotes bilayer reorganization, providing a new tool to understand ceramide effects on both model and cellular membranes.

\section{References:}

[1] S Zou and LJ Johnston, Curr. Opin. Coll. Inter. Sci. 15 (2010) p. 489.

[2] Y Zhang et al, Biochim. Biophys. Acta 1788 (2009) p. 178.

[3] Ira et al, J. Struct. Biol. 168 (2009) p. 78.

[4] DM Carter Ramirez, WW Ogilvie and LJ Johnston, Biochim. Biophys. Acta 1798 (2010) p. 558.

[5] YA Kim et al, Chem. Commun. 47 (2011) p. 9236.

[6] DM Carter Ramirez et al, Langmuir, in press.
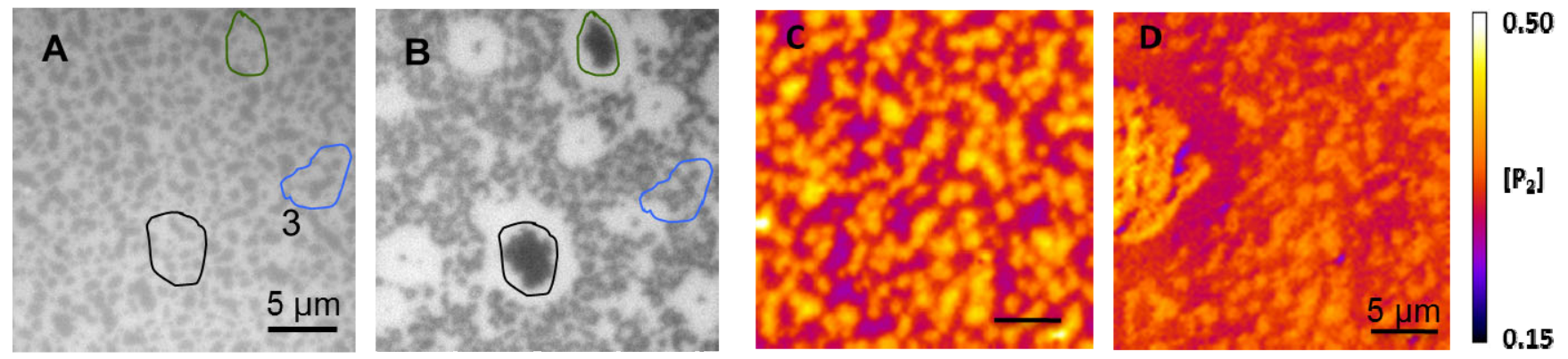

Figure 1. Fluorescence images showing a phase separated bilayer before (A) and after (B) enzyme treatment. Order parameter maps showing a similar bilayer before (C) and after (D) enzyme treatment.
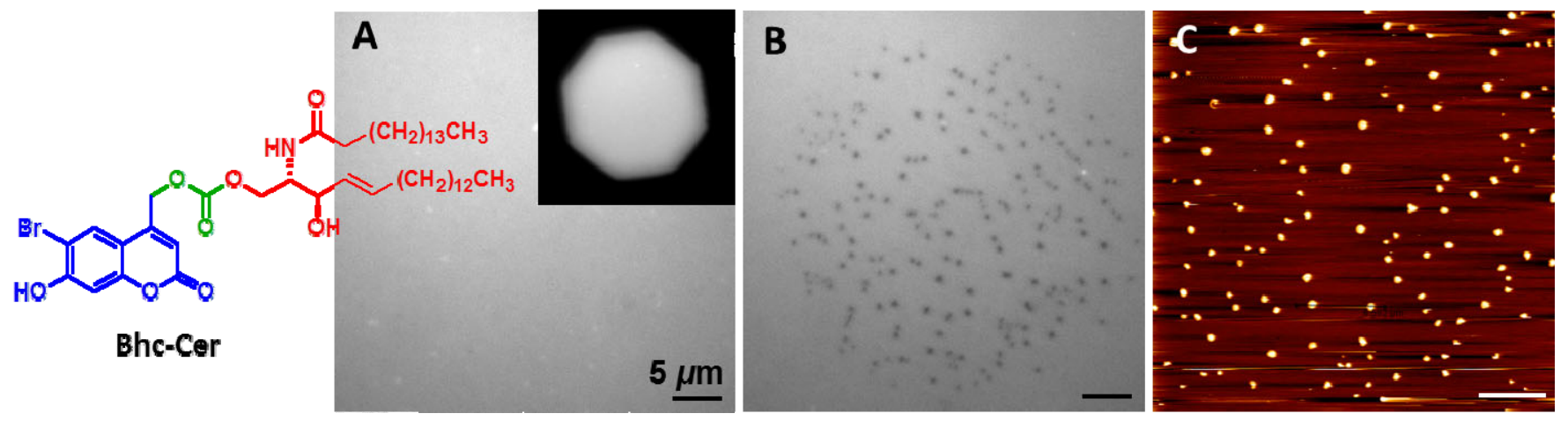

Figure 2. Structure of Bhc-Cer. Fluorescence $(A, B)$ and AFM (C) images showing a PC bilayer containing $10 \%$ Bhc-Cer before $(\mathrm{A})$ and after $(\mathrm{B}, \mathrm{C})$ photolysis to generate small ceramide domains. 